\title{
A Multistep Formulation of the Optimized Lax-Wendroff Method for Nonlinear Hyperbolic Systems in Two Space Variables
}

\author{
By A. R. Gourlay and J. L1. Morris
}

Introduction. In this note we develop a multistep formulation of the optimized Lax-Wendroff method for hyperbolic systems. This scheme was derived by Strang [6], [7]. The present formulation extends in a natural way, the two-step formulation of Richtmyer [5] for systems in one-space variable. We summarise this case in the next section.

One Space Dimension. We consider the first-order conservation law

$$
\partial u / \partial t+\partial f(u) / \partial x=0
$$

together with appropriate initial and boundary conditions. Let $A \equiv \partial f / \partial u$ denote the Jacobian matrix $A$ of the derivatives of the vector function $f$ with respect to the components of the unknown vector $u$.

The two-step Lax-Wendroff scheme for the numerical integration of (1) is given by

$$
\begin{aligned}
u_{i}^{*}{ }^{n+1} & =\frac{1}{2}\left(u_{i+1 / 2}^{n}+u_{i-1 / 2}^{n}\right)-(p / 2)\left(f_{i+1 / 2}^{n}-f_{i-1 / 2}^{n}\right), \\
u_{i}^{n+1} & =u_{i}^{n}-p\left(f_{i+1 / 2}^{* n+1}-f_{i-1 / 2}^{*^{n+1}}\right)
\end{aligned}
$$

where $u_{i}{ }^{n}=u(i h, n k), h, k$ being the mesh spacings in the space and time directions respectively, and where $p=k / h$. In (2) we regard $u_{i}{ }^{* n+1}$ as an intermediate value in the calculation.

For ease of writing we define the following finite-difference operators in the $x$ direction

$$
\mu_{x} u_{i}^{n} \equiv \mu_{x} u^{n}=\frac{1}{2}\left(u_{i+1 / 2}^{n}+u_{i-1 / 2}^{n}\right), \quad \delta_{x} u_{i}^{n}=u_{i+1 / 2}^{n}-u_{i-1 / 2}^{n} .
$$

Therefore (2) may be written in the form

$$
u^{* n+1}=\mu_{x} u^{n}-(p / 2) \delta_{x} f^{n}, \quad u^{n+1}=u^{n}-p \delta_{x} f^{*_{n+1}},
$$

where we have omitted lower suffices. If $\lambda$ is an eigenvalue of the Jacobian matrix $A$ then it is well known that (3) is stable (in the linearized sense) if $p|\lambda| \leqq 1$ (by von Neumann's criterion), and that it has second-order accuracy. A generalization of the scheme (3) is considered in Gourlay and Morris [3].

Two Space Dimensions. Consider now the two-dimensional conservation law

$$
\partial u / \partial t+\partial f(u) / \partial x+\partial g(u) / \partial y=0,
$$

together with suitable initial and boundary conditions. As in the preceding section we define $A \equiv \partial f / \partial u, B \equiv \partial g / \partial u$, and the difference operators $\mu_{y, y} \delta_{y}$ in the $y$-direc-

Received August 28, 1967. Revised November 13, 1967. 
tion. Then Richtmyer's two-step version [5] of the Lax-Wendroff scheme [4] is given by

$$
\begin{aligned}
u^{*^{n+1}} & =\frac{1}{2}\left(\mu_{x}+\mu_{y}\right) u^{n}-(p / 2)\left[\delta_{x} f^{n}+\delta_{y} g^{n}\right], \\
u^{n+1} & =u^{n}-p\left[\delta_{x} f^{*^{n+1}}+\delta_{y} g^{*^{n+1}}\right] .
\end{aligned}
$$

Scheme (5) is stable (in the linearized sense) if

$$
p\left|\lambda_{A}\right| \leqq(8)^{-1 / 2}, \quad p\left|\lambda_{B}\right| \leqq(8)^{-1 / 2},
$$

where $\lambda_{A}, \lambda_{B}$ are the eigenvalues of $A, B$ respectively. The stability restrictions (6) are much more severe than those of the one-dimensional Lax-Wendroff method. Scheme (5) again has second-order accuracy, and a generalization is considered in [3]. In order to alleviate such severe stability restrictions, several authors have considered modifications of scheme (5). However, such modifications usually require the evaluation of the Jacobian matrices $A, B$ and therefore the simple form of the two-step formulation is destroyed. We mention the work of Lax and Wendroff [4] and Burstein [1], [2], in this context. In [6], Strang showed how the best stability characteristics

$$
p\left|\lambda_{A}\right| \leqq 1, \quad p\left|\lambda_{B}\right| \leqq 1,
$$

for an explicit scheme, could be obtained. If we denote by $M_{x}$ the Lax-Wendroff difference operator in the $x$ direction, then Strang's scheme consists of using

$$
u^{n+1}=\frac{1}{2}\left[M_{x} M_{y}+M_{y} M_{x}\right] u^{n} .
$$

As pointed out by Burstein [1], this amounts to adding to the Lax-Wendroff operator in two dimensions the pseudo-viscous term

$$
\begin{aligned}
& -\frac{1}{8} p^{3}\left\{\left(A B^{2}+B^{2} A\right)\left(\Delta_{x}+\nabla_{x}\right) \Delta_{y} \nabla_{y}+\left(B A^{2}+A^{2} B\right)\left(\Delta_{y}+\nabla_{y}\right) \Delta_{x} \nabla_{x}\right\} u^{n}, \\
& +\frac{1}{8} p^{4}\left\{A^{2} B^{2}+B^{2} A^{2}\right\} \Delta_{x} \nabla_{x} \Delta_{y} \nabla_{y} u^{n},
\end{aligned}
$$

where $\Delta, \nabla$ are the usual forward and backward difference operators, and where we have assumed for ease that $A, B$ are constant matrices. Obviously the computation of such a term is time-consuming in the extreme. We shall now derive a multistep version of Strang's scheme which requires nothing more than the evaluation of the functions $f$ and $g$.

Multistep Strang Scheme. We consider first of all the linear system

$$
\partial u / \partial t+A \partial u / \partial x+B \partial u / \partial y=0,
$$

where $A$ and $B$ are constant matrices.

Strang's scheme is

$$
u^{n+1}=\frac{1}{2}\left[M_{x} M_{y}+M_{y} M_{x}\right] u^{n},
$$

where $M_{x}, M_{y}$ are Lax-Wendroff difference operators in the $x$ and $y$ directions respectively. We introduce the quantities $v_{(2)}^{n+1}$ and $w_{(2)}^{n+1}$ defined by the relations

$$
v_{(2)}^{n+1}=M_{y} u^{n}, \quad w_{(2)}^{n+1}=M_{x} u^{n},
$$

and the quantities $v_{(4)}^{n+1}$ and $w_{(4)}^{n+1}$ by means of

$$
v_{(4)}^{n+1}=M_{x} v_{(2)}^{n+1}, \quad w_{(4)}^{n+1}=M_{y} w_{(2)}^{n+1} .
$$


Then we have that

$$
u^{n+1}=\frac{1}{2}\left(v_{(4)}^{n+1}+w_{(4)}^{n+1}\right) .
$$

Each of the equations in (9) and (10) is "locally one-dimensional" in character and we therefore may employ the one-dimensional Richtmyer method (3) for each definition. For the nonlinear conservation law system

$$
\partial u / \partial t+\partial f(u) / \partial x+\partial g(u) / \partial y=0
$$

this leads to the following scheme:

$$
\begin{array}{rlrl}
v_{(1)}^{n+1}=\mu_{y} u^{n}-(p / 2) \delta_{y} g^{n}, & w_{(1)}^{n+1}=\mu_{x} u^{n}-(p / 2) \delta_{x} f^{n}, \\
v_{(2)}^{n+1}=u^{n}-p \delta_{y} g_{(1)}^{n+1}, & w_{(2)}^{n+1}=u^{n}-p \delta_{x} f_{(1)}^{n+1}, \\
v_{(3)}^{n+1}=\mu_{x} v_{(2)}^{n+1}-(p / 2) \delta_{x} f_{(2)}^{n+1}, & w_{(3)}^{n+1}=\mu_{y} w_{(2)}^{n+1}-(p / 2) \delta_{y} g_{(2)}^{n+1} \\
v_{(4)}^{n+1}=v_{(2)}^{n+1}-p \delta_{x} f_{(3)}^{n+1}, & w_{(4)}^{n+1}=w_{(2)}^{n+1}-p \delta_{y} g_{(3)}^{n+1}, \\
u^{n+1}=\frac{1}{2}\left(v_{(4)}^{n+1}+w_{(4)}^{n+1}\right) .
\end{array}
$$

It is straightforward to verify that (12), (13), (14) have second-order accuracy. (We note the notation $g^{n}=g\left(u^{n}\right), g_{(1)}^{n+1}=g\left(v_{(1)}^{n+1}\right)$ etc.)

Since the majority of computing time in such problems is taken in the evaluation of the vector functions $f$ and $g$ we will compare the algorithms on this basis. It is then easily seen that the multistep algorithm requires roughly twice as much computing time as does the Lax-Wendroff method (5), but the stability characteristics of (12), (13), (14) are $\sqrt{ } 8$ times better than those of the method (5). Apart from this advantage, the multistep Strang method is very easy to program, requiring only a single procedure equivalent to a one-dimensional Lax-Wendroff algorithm. Moreover, if it is required to add in a pseudo-viscous term, this merely takes its normal place in the respective one-dimensional algorithm and will itself be a one-dimensional quantity. Therefore the results (empirical or otherwise) obtained for scheme (3) may be carried through to two space dimensions with ease.

It would appear therefore that the multistep formulation of Strang's scheme has a great deal to offer in its simplicity and its stability.

Generalizations. We briefly note a few extensions of the above results to more general first-order systems. The two-step Lax-Wendroff method for the system

$$
\partial u / \partial t+\partial f(x, t, u) / \partial x=z(x, t, u)
$$

may be written in the form,

$$
\begin{aligned}
& u_{(1)}^{n+1}=\mu_{x} u^{n}-(p / 2)\left[\delta_{x} \bar{f}^{n}-h \bar{z}^{n}\right], \\
& u^{n+1}=u^{n}-p\left[\delta_{x} \bar{f}_{(1)}^{n+1}-h \bar{z}_{(1)}^{n+1}\right]
\end{aligned}
$$

where $h$ is the mesh spacing in the space direction and where

$$
\bar{f}^{n} \equiv f\left(x,\left(n+\frac{1}{2}\right) k, u^{n}\right), \quad \bar{f}_{(1)}^{n+1} \equiv f\left(x,\left(n+\frac{1}{2}\right) k, u_{(1)}^{n+1}\right), \text { etc. }
$$

Therefore, for the two-dimensional system

$$
\partial u / \partial t+\partial f(x, y, t, u) / \partial x+\partial g(x, y, t, u) / \partial y=z(x, y, t, u),
$$


we have the multistep Strang scheme:

$$
\begin{array}{ll}
v_{(1)}^{n+1}=\mu_{y} u^{n}-(p / 2)\left[\delta_{y} \bar{g}^{n}-h \bar{q}^{n}\right], & w_{(1)}^{n+1}=\mu_{x} u^{n}-(p / 2)\left[\delta_{x} \bar{f}^{n}-h \bar{s}^{n}\right], \\
v_{(2)}^{n+1}=u^{n}-p\left[\delta_{y} \bar{g}_{(1)}^{n+1}-h \bar{q}_{(1)}^{n+1}\right], & w_{(2)}^{n+1}=u^{n}-p\left[\delta_{x} \bar{f}_{(1)}^{n+1}-h \bar{s}_{(1)}^{n+1}\right], \\
v_{(3)}^{n+1}=\mu_{x} v_{(2)}^{n+1}-(p / 2)\left[\delta_{x} \bar{f}_{(2)}^{n+1}-h \bar{s}_{(2)}^{n+1}\right], & w_{(3)}^{n+1}=\mu_{y} w_{(2)}^{n+1}-(p / 2)\left[\delta_{y} \bar{g}_{(2)}^{n+1}-h \bar{q}_{(2)}^{n+1}\right] \\
v_{(4)}^{n+1}=v_{(2)}^{n+1}-p\left[\delta_{x} \bar{f}_{(3)}^{n+1}-h \bar{s}_{(3)}^{n+1}\right], & w_{(4)}^{n+1}=w_{(2)}^{n+1}-p\left[\delta_{y} \bar{g}_{(3)}^{n+1}-h \bar{q}_{(3)}^{n+1}\right], \\
u^{n+1}=\frac{1}{2}\left(v_{(4)}^{n+1}+w_{(4)}^{n+1}\right),
\end{array}
$$

where

$$
z(x, y, t, u)=q(x, y, t, u)+s(x, y, t, u) .
$$

It is somewhat surprising that an arbitrary splitting of $z$ in the form (16) maintains second-order accuracy of the above scheme. Notice finally that we may even allow $z$ to include second-order derivatives $u_{x x}, u_{y y}, u_{x y}$, and thus we can write the full Navier-Stokes equations in the form (15) (see Thommen [8]).

A numerical example. A simple comparison of the multistep Strang scheme and Richtmyer's two-step Lax-Wendroff scheme [5] was undertaken for the problem

$$
\frac{\partial u}{\partial t}+\frac{\partial}{\partial x}\left(\frac{1}{4} u^{2}\right)+\frac{\partial}{\partial y}\left(\frac{1}{4} u^{2}\right)=z(u, x, y),
$$

where

$$
z(u, x, y)=50 u x y[y(1-y)(2-3 x)+x(1-x)(2-3 y)],
$$

subject to the initial condition

$$
u(x, y, 0)=100 x^{2}(1-x) y^{2}(1-y)
$$

and zero boundary conditions on $[0 \leqq x, y \leqq 1] \times[0 \leqq t \leqq T]$. This problem has the time-independent solution

$$
u(x, y, t)=100 x^{2} y^{2}(1-x)(1-y) .
$$

It may be easily verified that the maximum value of the function $u(x, y, t)$ is approximately 2.2 . It follows that the stability conditions for the schemes are approximately as follows:

Two-step Lax-Wendroff: $p \leqq .32$,

Multistep Strang: $p \leqq .90$.

In practice it was found that the multistep Strang scheme did become unstable for $p>.90$ but that the two-step Lax-Wendroff scheme remained stable for $p<.60$. This result is in agreement with previous authors who have noted that the LaxWendroff stability characteristics are too severe. If such a result is typical, the LaxWendroff scheme would appear to be competitive with the multistep Strang formulation. However, the case of programming of the latter scheme, and the ability to incorporate pseudo-viscous terms in a one-dimensional manner, must make it more attractive in practice. 
1. S. Z. Burstein, "Numerical calculations of multidimensional shocked flows," AIAA J., v. 2, 1964, pp. 2111-2117. MR 31 \#4304.

2. S. Z. Burstein, "Finite difference calculations for hydrodynamic flows containing discontinuities," J. Computational Phys., v. 1, 1966, pp. 198-222.

3. A. R. GourlaY \& J. L.. MoRRIS, "Finite-difference methods for nonlinear hyperbolic systems," Math. Comp., v. 22, 1968, pp. 28-39.

4. P. D. LAX \& B. WENDROFF, "Difference schemes for hyperbolic equations with high order of accuracy," Comm. Pure Appl. Math., v. 17, 1964, pp. 381-398. MR 30 \#722.

5. R. I. Richtmyer, A Survey of Difference Methods for Non-Steady Fluid Dynamics, N.C.A.R. Tech. Notes $63-2,1963$.

6. W. G. StRANG, "Accurate partial difference methods. I: Linear Cauchy problems," Arch. Rational Mech. Anal., v. 12, 1963, pp. 392-402. MR 26 \#4489.

7. W. G. STRANG, "Accurate partial difference methods. II: Nonlinear problems," Numer. Math., v. 6, 1964, pp. 37-46. MR $29 \# 4215$.

8. H. V. Thommen, A Method for the Numerical Solution of the Complete Navier-Stokes Equatims for Steady Flows, General Dynamics/Astronautics GDC-ERR-AN 733, April 1965. 